\title{
Long-Term Outcomes of Group B Eyes in Patients with Retinoblastoma Treated with Short-Course Chemoreduction: Experience from Children's Hospital Los Angeles/University of Southern California
}

\author{
Dagny Zhu ${ }^{\text {a }}$ Jesse L. Berry ${ }^{a, c}$ Lilangi Ediriwickrema ${ }^{\text {a }}$ Kenneth Wong ${ }^{d}$ \\ Thomas C. Lee ${ }^{a, c}$ A. Linn Murphree ${ }^{c}$ Jonathan W. Kim ${ }^{a, c}$ Rima Jubran ${ }^{b}$ \\ a University of Southern California Eye Institute, b The Children's Center for Cancer and Blood Diseases, Children's Hospital, \\ 'The Vision Center at Children's Hospital Los Angeles, and ' Radiation Oncology Program, Children's Hospital, \\ Los Angeles, Calif., USA
}

\section{Key Words}

Retinoblastoma · Pediatric oncology · Chemotherapy

\begin{abstract}
Background/Aims: Chemoreduction protocols for retinoblastoma vary widely across institutions. Herein, we compare a 3-versus 6-cycle chemotherapy approach for group B retinoblastoma. Methods: A nonrandomized, retrospective review of patients diagnosed with group $B$ retinoblastoma from 1991-2011 at Children's Hospital Los Angeles was performed. A total of 72 eyes of 63 patients were analyzed. Mean follow-up time was 82 months (range 6-272 months). Main outcome measures were globe salvage and need for external beam radiation. Results: Forty-six patients (55 eyes) were treated upfront with 3 cycles of carboplatin, etoposide, and vincristine; 17 patients ( 17 eyes) received 6 cycles. Thirty-seven eyes (67\%) in the 3-cycle group were cured with initial chemoreduction alone. An additional 10 eyes with persistent or recurrent tumors were rescued with 3 more cycles for a total salvage rate of $85 \%$ ( $47 / 55$ eyes). In the 6 -cycle group, 16 of 17 eyes (94\%) avoided radiation and enucleation. Conclusion: The initial recurrence rate was higher for the 3-cycle group $(p=0.03)$. However, eyes failing short-course chemoreduction were rescued with 3 additional cycles and achieved
\end{abstract}

a similar overall event-free survival rate $(p=0.16)$. In our cohort, this short-course approach spared 63\% (29/46) of patients with group B retinoblastoma the extra 3 cycles of systemic chemotherapy.

(c) 2015 S. Karger AG, Basel

\section{Introduction}

Treatment of retinoblastoma using chemotherapy was first introduced in the early 1960 s $[1,2]$. Since then, a variety of chemotherapy regimens have been described, but none abandoned the concomitant use of radiation therapy until 1996 [3-6]. These studies led to the modern era of treatment with primary chemoreduction and concurrent local consolidative therapies, reserving external beam radiation for refractory disease [7]. In the last decade, local or regional delivery of chemotherapy to patients with retinoblastoma including intra-arterial and intravitreal approaches has also been described and implemented globally [8-13].

In 2003, the International Classification of Retinoblastoma grouped intraocular retinoblastoma based on tumor size, location, extent of seeding, and retinal detachment (groups A-E; online suppl. table 1; see www.

\section{KARGER}

E-Mail karger@karger.com

www.karger.com/oop (c) 2015 S. Karger AG, Basel

2296-4681/15/0022-0105\$39.50/0
Dagny Zhu, MD

University of Southern California Eye Institute

1450 San Pablo Street

Los Angeles, CA 90033 (USA)

E-Mail dagny.zhu@gmail.com 
karger.com/doi/10.1159/000439593 for all online suppl. material) [14]. The success of chemoreduction varies with the stage of disease, with groups $\mathrm{A}-\mathrm{C}$ reportedly achieving cure rates as high as $90-100 \%$ [15-18]. The globe preservation rates for group $\mathrm{B}$, in particular, vary widely from 70 to $100 \%$ due to inherent differences between chemoreduction protocols across institutions [1922 ]. Despite relatively high overall salvage rates for group $B$ eyes, the ideal chemoreduction protocol remains unclear. Currently, many institutions use 6 cycles of systemic carboplatin, etoposide, and vincristine (CEV) as the first-line treatment modality for group B-D retinoblastoma [5-7, 15]. At Children's Hospital Los Angeles (CHLA), an overall higher dose of carboplatin is given over 2 days for all patients receiving chemoreduction (13 $\mathrm{mg} / \mathrm{kg} \times 2$ days), and for group B eyes, a short-course 3 -cycle chemoreduction protocol is employed. In this study, we evaluated outcomes of group B retinoblastoma eyes treated at CHLA with a short-course 3-cycle versus 6-cycle chemotherapy approach.

\section{Methods}

A nonrandomized, comparative retrospective review with data collection spanning 20 years from January 1, 1991 to December 31, 2011 at CHLA was performed. The Institutional Review Board at CHLA approved this study.

Patients diagnosed at CHLA with retinoblastoma and designated group B in at least 1 eye from January 1, 1991 to December 31, 2011 at CHLA were included in this study. Patients with evidence of extraocular disease at the time of diagnosis were treated according to the Children's Oncology Group protocol for infants with central nervous system disease and were thus excluded from analysis. Patients treated with systemic chemoreduction protocols other than the standard CHLA group B treatment protocol described below were excluded. Those treated under different protocols at outside hospitals and referred for second opinions were also excluded.

\section{Treatment}

The treatment protocol for group B retinoblastoma eyes at CHLA is systemic chemoreduction and local consolidation therapy, which includes argon $(532 \mathrm{~nm})$ or diode laser therapy $(810 \mathrm{~nm}$ laser), cryotherapy (freeze-thaw cycle $\times 2$ ) for larger lesions anterior to the equator, and, rarely, radioactive plaque. The systemic chemoreduction protocol is summarized in online supplementary table 2 and includes intravenous carboplatin $390 \mathrm{mg} / \mathrm{m}^{2}(13 \mathrm{mg} /$ $\mathrm{kg}$ for children $<36$ months $) \times 2$ days, etoposide $100 \mathrm{mg} / \mathrm{m}^{2}(5 \mathrm{mg} /$ $\mathrm{kg}$ for children $<36$ months) $\times 2$ days, and vincristine $1.5 \mathrm{mg} / \mathrm{m}^{2}$ $(0.05 \mathrm{mg} / \mathrm{kg}$ for children $<36$ months $) \times 1$ day. Infants $<6$ months of age at diagnosis receive a modified dosing regimen with a $50 \%$ decrease in all agents for the first cycle [23]. At our institution, vincristine is routinely omitted for patients $<2$ months of age due to concerns regarding paralytic ileus and irritability. After the first cycle at $50 \%$ dose CEV (or CE), patients are then monitored for intraocular tumor response and grade III systemic toxicity. If there is adequate tumor response or evidence of systemic toxicity, patients are kept at the $50 \%$ dose for the next cycle. If there is inadequate tumor response and no grade III toxicity, then the dose is increased to $75 \%$. Patients can be increased to $100 \%$ dose of CEV if all factors are present: (1) older than 3 months of age, (2) no grade III toxicity to $75 \%$ dose, and (3) inadequate tumor response to $75 \%$ dose.

Unilateral group B cases or bilateral cases in which the group B eye was the most advanced eye received an initial 3 cycles of highdose $\mathrm{CEV}$ to treat the eye. In bilateral cases wherein the group $\mathrm{B}$ eye was the least advanced eye, 6 cycles of the same high-dose CEV regimen was given upfront based on the staging of the more affected eye (group C or D). In cases where the most advanced eye was enucleated, the total number of cycles was dependent on the histopathology of the enucleated eye. If high-risk features such as postlaminar optic nerve invasion or massive choroidal invasion were found, 3 additional cycles of adjuvant chemotherapy were given. Since adjuvant therapy utilizes a slightly lower dose of carboplatin $(18.6 \mathrm{mg} / \mathrm{kg})$, these cases were excluded from the analysis (online suppl. table 2).

After completion of initial chemoreduction, patients in the short-course 3-cycle group were referred for additional chemoreduction ( 3 more cycles of high-dose CEV) if there was tumor persistence or recurrence not amenable to local control. Rarely, patients also required intensity-modulated radiation therapy (IMRT) for salvage at a dose of $36 \mathrm{~Gy}$ (for all but 2 patients who were prescribed 44 and 26 Gy). Patients were examined at intervals of 4-6 weeks during treatment.

\section{Chart Review}

At initial evaluation, each patient had a full staging exam under anesthesia (EUA) including B-scan ultrasound evaluation. Eyes were classified according to the International Classification System for Retinoblastoma [14]. A few eyes that had been initially classified under the older Reese-Ellsworth system were reassigned according to the International Classification System for Retinoblastoma based on fundus photos and documentation of exam findings. A retrospective chart review was done to obtain the following information: date of birth, date of diagnosis, gender, laterality of retinoblastoma, length of follow-up, systemic chemotherapy agents used with number of cycles, radiation therapy when applicable, and details of local therapy and visual acuity of the treated eye(s) at the last follow-up visit. Complications of therapy including National Cancer Institute common terminology criteria for adverse events (CTCAE) version 3, grade 3 or higher, additional tumors, and deaths were also recorded.

\section{Statistical Analysis}

Mean, median, and standard deviations were computed using Microsoft Excel functions. $p$ values were calculated with the $\chi^{2}$ function and 2-tailed T test. Kaplan-Meier curves were calculated with Microsoft Excel. Vassarstats.net was used to obtain the confidence intervals for both cohorts.

\section{Images}

Images were obtained using a wide-angle contact fundus camera (RetCam II, Clarity Medical Systems, Inc., Pleasanton, Calif., USA) and ultrasound during EUA. 


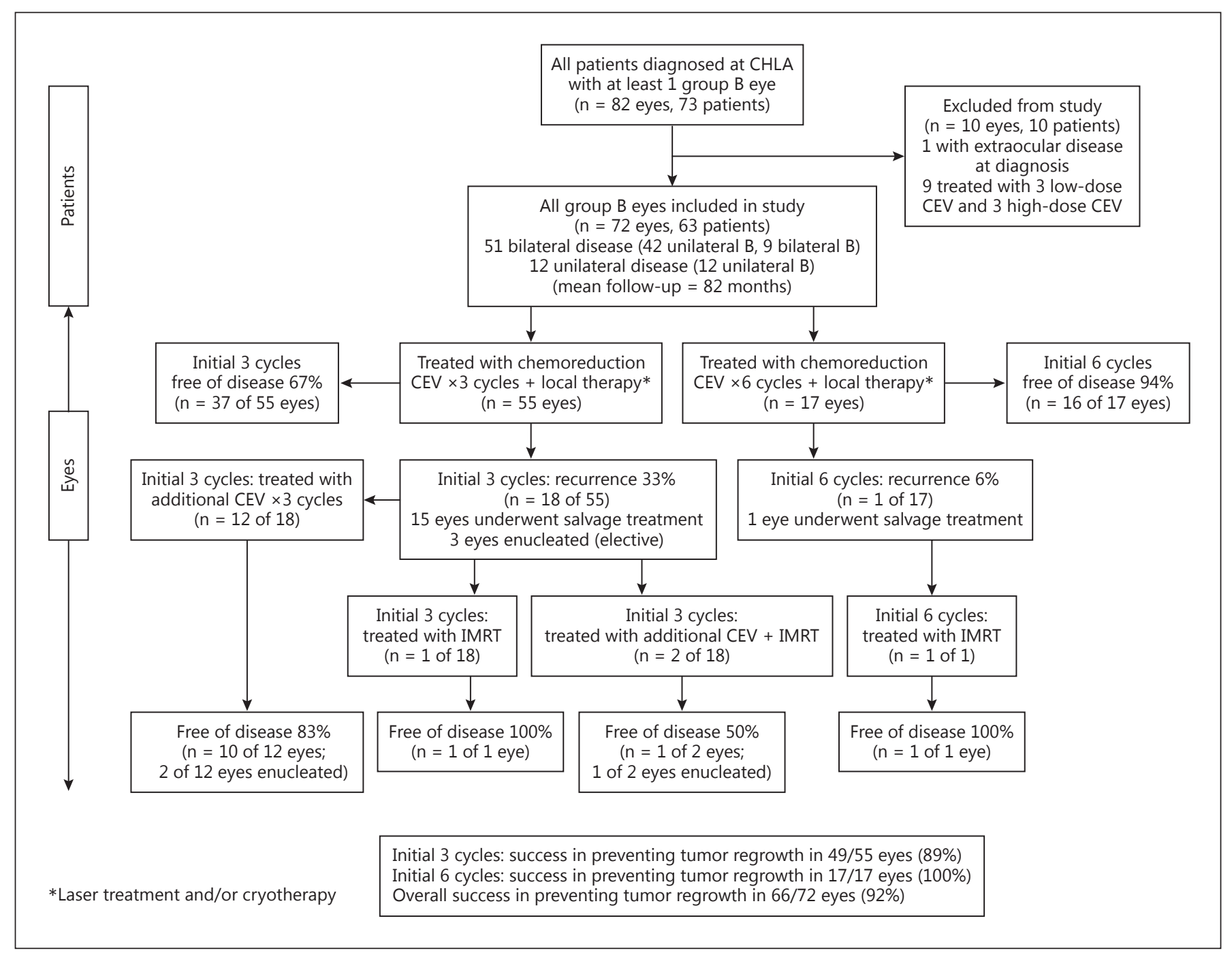

Fig. 1. Modified CONSORT diagram showing the progress of patients through the study [24, 25].

\section{Results}

From January 1, 1991 to December 31, 2011, 740 patients were referred to CHLA for retinoblastoma. Eightytwo eyes of 73 patients were diagnosed with group B retinoblastoma during EUA. One patient was excluded due to the presence of extraocular disease at the time of diagnosis and was thus treated under a different protocol. Another 9 patients with contralateral group $\mathrm{E}$ eyes with high-risk pathology treated with a heterogeneous combination of 6-cycle CEV (3 high-dose and 3 low-dose) were also excluded. Therefore, a total of 63 patients were included in the analysis. Of these, 51 patients had bilateral retinoblastoma of which 42 patients had 1 group $B$ eye and 9 had bilateral group B disease (60 total group B eyes included). Twelve patients had unilateral group B disease. Therefore, a total of 72 group B eyes of 63 patients were included in this study. A modified Consolidated Standards of Reporting Trials (CONSORT) flow diagram is shown in figure $1[24,25]$.

Patient characteristics are listed in table 1. Mean age at diagnosis was 6.3 months (range 0.7-26.7 months). The majority of patients were diagnosed at $<6$ months of age (59\%). In $48 \%(30 / 63)$ of patients, group B was the most advanced eye followed by group E (27\%), group D (17\%), and group $\mathrm{C}(8 \%)$. The mean follow-up time for all patients was 82 months (range 6-272 months). 
Table 1. Patient characteristics

\begin{tabular}{|c|c|c|c|}
\hline & $\begin{array}{l}\text { All patients } \\
(\mathrm{n}=63)\end{array}$ & $\begin{array}{l}3 \text {-cycle regimen } \\
(\mathrm{n}=46)\end{array}$ & $\begin{array}{l}\text { 6-cycle regimen } \\
(\mathrm{n}=17)\end{array}$ \\
\hline \multicolumn{4}{|l|}{ Age at diagnosis } \\
\hline$<6$ months & $37(59 \%)$ & - & - \\
\hline $6-12$ months & $20(32 \%)$ & - & - \\
\hline $12-18$ months & $5(8.0 \%)$ & - & - \\
\hline$>18$ months & $1(1.6 \%)$ & - & - \\
\hline \multicolumn{4}{|c|}{ Mean age at diagnosis, months } \\
\hline Overall & 6.3 & 6.0 & 7.2 \\
\hline Unilateral & 9.5 & - & - \\
\hline Bilateral & 5.5 & - & - \\
\hline \multicolumn{4}{|l|}{ Gender } \\
\hline Male & $37(59 \%)$ & $23(50 \%)$ & $14(82 \%)$ \\
\hline Female & $26(41 \%)$ & $23(50 \%)$ & $3(18 \%)$ \\
\hline \multicolumn{4}{|c|}{ Laterality of retinoblastoma } \\
\hline Unilateral & $12(19 \%)$ & $12(26 \%)$ & 0 \\
\hline Bilateral & $51(81 \%)$ & $34(74 \%)$ & $17(100 \%)$ \\
\hline \multicolumn{4}{|c|}{ Stage of most advanced eye at time of diagnosis } \\
\hline $\mathrm{B}$ & $30(48 \%)$ & $30(65 \%)$ & 0 \\
\hline $\mathrm{C}$ & $5(7.9 \%)$ & 0 & $5(29 \%)$ \\
\hline $\mathrm{D}$ & $11(17 \%)$ & $3(6.5 \%)^{\mathrm{a}}$ & $8(47 \%)$ \\
\hline $\mathrm{E}$ & $17(27 \%)$ & $13(28 \%)$ & $4(24 \%)$ \\
\hline
\end{tabular}

${ }^{a}$ Patients were treated with 3 instead of 6 initial cycles because the contralateral eye was later enucleated and found to have no high-risk pathology.

\section{Initial Chemoreduction with Short-Course 3-Cycle} versus 6-Cycle Therapy

To evaluate the outcomes of chemoreduction as primary treatment for group B eyes, the data was divided into 2 groups: those eyes treated with 3 initial cycles of chemoreduction versus those treated with 6 cycles. All group $B$ patients with unilateral disease or in which group $\mathrm{B}$ was the most advanced eye received 3 cycles of chemoreduction upfront according to the CHLA protocol (online suppl. table 2). Overall, 55 eyes of 46 patients were treated with the short-course 3-cycle approach. This group of 55 group B eyes also included bilateral patients who had the more advanced eye (group D or E) enucleated but did not receive adjuvant chemotherapy. Standard 6-cycle chemoreduction was given to 17 group B eyes of 17 patients based on the staging of the more advanced contralateral eye.

Primary cure rates were higher and recurrence rates lower for those treated initially with 6 cycles compared to 3 cycles of CEV (fig. 1). Specifically, 16 of 17 (94\%) group $\mathrm{B}$ eyes were cured with $6 \mathrm{CEV}$ cycles, while only 37 of 55 (67\%) group B eyes were cured with $3 \mathrm{CEV}$ cycles (plus local consolidation therapy). This difference was statistically significant $(p=0.03)$. Of the 18 eyes that failed initial treatment with 3 cycles of CEV, 15 (83\%) were treated with additional chemoreduction and/or IMRT with relatively high success (fig. 1). Twelve of the 14 patients (86\%) who underwent additional chemoreduction received those cycles within 6 months of initial treatment. For details regarding the breakdown of salvage treatment, see figure 1 .

Overall, for the 55 group B eyes treated initially with 3 cycles of CEV (including those that received additional cycles as needed), the event-free globe survival rate (avoiding radiation or enucleation) was 85\% (47/55 eyes). This approached the event-free survival rate for the initial 6 -cycle chemoreduction group ( $94 \%$ or $16 / 17$ eyes). The final globe preservation rate of group B eyes treated with chemoreduction plus IMRT as salvage was $89 \%$ (49 of 55 eyes) for those treated with 3 cycles upfront and 100\% (17 of 17 eyes) for those treated with an initial 6 cycles. At the end of treatment, there was no statistically significant difference in the treatment outcomes between the 2 groups $(\mathrm{p}=0.16)$. At 60 months, the Kaplan-Meier estimate of globe salvage after chemoreduction alone (e.g. no IMRT) for the short-course 3-cycle group was $87.0 \%$ (95\% confidence interval 74.6-94.1\%), and for the 6-cycle group was $94.1 \%$ (95\% confidence interval 69.2-99.7\%; online suppl. fig. 1).

Of all the 72 group B eyes treated in this study, 19 (26\%) failed initial chemoreduction therapy and were offered additional cycles of chemoreduction and/or IMRT as salvage therapy. Of these, 13 eyes (68\%) were ultimately preserved. Overall, 66 of 72 (92\%) group B eyes avoided enucleation and 63 of 72 (88\%) group B eyes avoided enucleation and radiation altogether (fig. 1).

\section{Toxicity and Secondary Malignancies}

Grade 3 or higher CTCAE febrile neutropenia is defined as fever of unknown origin, without clinically documented infection, with an absolute neutrophil count $<1.0$ $\times 10^{9} / 1$ and fever $>38.5^{\circ} \mathrm{C}$. In this study, 3 of $55(5.5 \%)$ patients treated with 3 -cycle chemoreduction developed febrile neutropenia versus 7 of 17 (41\%) patients who had been treated with 6 cycles. None of the patients developed hearing loss. Two patients were later diagnosed with pineoblastoma (both diagnosed initially with bilateral disease), one of whom died 2.5 years after chemoreduction. No other patient developed a secondary solid malignancy, and there were no cases of metastatic disease during the mean follow-up period of 82 months (range 6-272 months). 


\section{Final Visual Acuity}

Of the 72 treated group B eyes, 28 (39\%) had a visual acuity of $20 / 25$ or better and 37 (52\%) had vision better than $20 / 50$ at their last follow-up visit. Many patients were too young at their last follow-up visit to have their vision quantitatively assessed and were only able to fix and follow (19/72-26\%; see online suppl. table 3 for further breakdowns).

\section{Discussion}

The success of using systemic chemotherapy regimens with local consolidative therapy for the treatment of intraocular retinoblastoma was initially adopted in the mid-1990s and is today widely accepted as a first-line treatment $[4-6,26]$. However, specific chemotherapy regimens appear to vary between centers $[5,16,19-22$, 27-31]. In 1996, Gallie et al. [5] reported treatment outcomes using a chemoreduction protocol consisting of 3-12 cycles of carboplatin, vincristine-teniposide, plus cyclosporine with relative success. The Children's Oncology Group (ARET0331) began a clinical trial using only 2 agents, vincristine and low-dose carboplatin, for group B tumors, but the study was prematurely closed. In 2003, Rodriguez-Galindo et al. [27] also studied a 2-agent protocol using 8 cycles of carboplatin and vincristine and reported an event-free survival estimate at 2 years of $59.2 \pm$ $12.0 \%$ for Reese-Ellsworth group I, II, and III eyes. Radiation therapy was required to increase the salvage rate to $83.3 \%$. In 2006, Shields et al. [16] reported treatment outcomes for group B-D eyes using a 6-cycle CEV regimen that incorporates a lower dose of carboplatin (18.6 $\mathrm{mg} / \mathrm{kg} \times 1$ day) to treat intraocular disease. Under this protocol, 93\% of group B eyes were preserved with chemoreduction. Reports of chemotherapy regimens utilizing less than 6 cycles for group B retinoblastoma in the literature are sparse. Bartuma et al. [28] reported an $80 \%$ overall success rate using 4-6 cycles of CEV for their patients with hereditary group B retinoblastoma. A few groups have reported the outcomes of using 2-4 chemotherapy cycles, but the majority of those patients analyzed were classified under the older Reese-Ellsworth system [29-31]. We are not aware of any institution-based 3-cycle chemoreduction protocols published in the literature for group B eyes.

In this study, we assessed the outcomes of 72 eyes of 63 patients with group B retinoblastoma treated at CHLA over a 20-year period and demonstrated an overall high success rate with our approach. Our overall globe preser-

Treatment Outcomes of Group B

Retinoblastoma Eyes vation rate of $88 \%$ (63/72 eyes) for group B eyes using CEV and local consolidation is similar to the $93 \%$ reported by Shields et al. [16]. Kaplan-Meier estimation of event-free survival (e.g. avoiding IMRT or enucleation) at 60 months posttreatment was relatively equal for both groups of patients treated with either an initial 3 cycles of chemoreduction ( \pm additional cycles as needed) or an initial 6 cycles ( 87 vs. 94\%, respectively). Therefore, there was no statistically significant difference in overall outcome between the short-course and standard chemoreduction groups at the end of therapy.

It is important to note that there were indeed fewer recurrences when treating group B eyes with 6 rather than 3 cycles of chemoreduction upfront (6 vs. $33 \%$, respectively). This highlights two important points: First, the use of additional chemoreduction for recurrences as needed was successful and improved the overall event-free survival rate from $67 \%$ ( $37 / 55$ eyes) to $85 \%$ ( $47 / 55$ eyes). This final globe survival rate for the short-course 3-cycle group was statistically similar to the $94 \%$ rate ( $16 / 17$ eyes) achieved by the 6-cycle group after chemoreduction alone (e.g. without IMRT as salvage). Of note, 12 of the 14 eyes ( $86 \%$ ) that required further chemoreduction received those additional cycles within 6 months of initial treatment suggesting relatively early identification of tumor persistence and/or recurrence. Given the well-known associated risks of radiation therapy including retardation of orbital bone growth $[32,33]$ and increased secondary malignancies [34-40], we recommend the use of additional chemoreduction prior to IMRT for tumors refractory to initial short-course 3-cycle chemoreduction.

Second, and more importantly, we observed that the initial 3-cycle regimen spared 63\% (29/46) of patients the extra 3 cycles of chemotherapy without affecting the longterm salvage rate. Using this short-course approach, some patients not only forgo the burden of undergoing a longer treatment course, but also enjoy a lower degree of exposure to carboplatin and etoposide. This may minimize the risk of developing dose-dependent toxicities related to platinum and epipodophyllotoxin-based chemotherapeutic agents. In our study, a significantly greater proportion of patients treated with 6-cycle CEV (41\%) developed febrile neutropenia compared to those treated with 3 total cycles (5.5\%). None of our patients developed significant organ dysfunction such as hearing loss. Two patients were diagnosed with pineoblastoma after their initial diagnosis of intraocular retinoblastoma, one of whom died 2.5 years after chemoreduction therapy. Both patients had received 6 cycles of high-dose CEV based on the status of their non-group B eye. 
The main limitation of this study is the retrospective nature of the data collection and analysis. In addition, the higher-dose carboplatin $(13 \mathrm{mg} / \mathrm{kg} \times 2$ days $)$ regimen used to treat group B retinoblastoma eyes at CHLA is different from the standard lower-dose 6-cycle carboplatin $(18.6 \mathrm{mg} / \mathrm{kg} \times 1$ day) regimen used at many institutions, and therefore, the results cannot be compared directly. Nevertheless, our short-course approach using rescue chemotherapy as needed achieved overall preservation rates comparable to those reported in the literature for group B eyes. Moreover, it should also be noted that $59 \%$ of the patients in this series were diagnosed before 6 months of age and received a lower dose regimen for at least a part of their course. Another limitation of this study is the relatively small number of patients in the 6 -cycle chemoreduction group. Nine patients treated with 6 cycles consisting of both high- and low-dose CEV were excluded in order to maintain consistency and minimize confounders as described above.

This study demonstrates the validity and efficacy of using a short-course 3-cycle chemoreduction approach for patients with unilateral group B eyes or bilateral disease wherein the group B eye is the most advanced eye. Tumors refractory to initial therapy can be effectively treated with an additional 3 cycles of chemoreduction without lowering overall success rates. This short-course approach using additional cycles only as needed achieves equal overall salvage rates as standard 6-cycle chemotherapy. While the additional long-term risks of using 6 chemotherapy cycles were limited in this study, our protocol spares a significant number of patients the potentially greater morbidity and risk of dose-dependent side effects known to be associated with chemotherapy.

\section{Acknowledgements}

The authors would like to acknowledge Kathleen McGovern, MS from The Vision Center at CHLA, Los Angeles, California, for her statistical expertise. No compensation was given.

\section{Statement of Ethics}

All research was performed under the guidelines for human studies. The Institutional Review Board at CHLA approved the study protocol. No patient informed consent was obtained given the retrospective nature of this study.

\section{Disclosure Statement}

There are no conflicts to disclose from any of the authors. Grant support was received from Retinoblastoma International, Inc., The Institute for Families, Inc., CHLA, and Research to Prevent Blindness.

\section{References}

1 Ericson LA, Rosengren BH: Tests of intravitreous chemotherapy in retinoblastoma. Acta Ophthalmol (Copenh) 1962;40:121-122.

2 Pratt CB: Topical topic. Use of chemotherapy for retinoblastoma. Med Pediatr Oncol 1998; 31:531-533.

3 White L: Chemotherapy for retinoblastoma: where do we go from here? A review of published literature and meeting abstracts, including discussions during the Vth International Symposium on Retinoblastoma, October 1990. Ophthalmic Paediatr Genet 1991; 12:115-130.

4 Murphree AL, Villablanca JG, Deegan WF 3rd, Sato JK, Malogolowkin M, Fisher A, Parker R, Reed E, Gomer CJ: Chemotherapy plus local treatment in the management of intraocular retinoblastoma. Arch Ophthalmol 1996;114:1348-1356.

5 Gallie BL, Budning A, DeBoer G, Thiessen JJ, Koren G, Verjee Z, Ling V, Chan HS: Chemotherapy with focal therapy can cure intraocular retinoblastoma without radiotherapy. Arch Ophthalmol 1996;114:1321-1328.
6 Shields CL, De Potter P, Himelstein BP, Shields JA, Meadows AT, Maris JM: Chemoreduction in the initial management of intraocular retinoblastoma. Arch Ophthalmol 1996;114:1330-1338.

7 Shields CL, Mashayekhi A, Cater J, Shelil A, Meadows AT, Shields JA: Chemoreduction for retinoblastoma: analysis of tumor control and risks for recurrence in 457 tumors. Trans Am Ophthalmol Soc 2004;102:35-44; discussion $44-45$.

8 Suzuki S, Yamane T, Mohri M, Kaneko A: Selective ophthalmic arterial injection therapy for intraocular retinoblastoma: the long-term prognosis. Ophthalmology 2011;118:20812087.

9 Gobin YP, Dunkel IJ, Marr BP, Brodie SE, Abramson DH: Intra-arterial chemotherapy for the management of retinoblastoma: fouryear experience. Arch Ophthalmol 2011;129: 732-737.

10 Shields CL, Bianciotto CG, Jabbour P, Ramasubramanian A, Lally SE, Griffin GC, Rosenwasser R, Shields JA: Intra-arterial chemo- therapy for retinoblastoma: report No. 1, control of retinal tumors, subretinal seeds, and vitreous seeds. Arch Ophthalmol 2011;129: 1399-1406.

11 Abramson DH, Marr BP, Dunkel IJ, Brodie S, Zabor EC, Driscoll SJ, Gobin YP: Intra-arterial chemotherapy for retinoblastoma in eyes with vitreous and/or subretinal seeding: 2-year results. Br J Ophthalmol 2012;96:499-502.

12 Vajzovic LM, Murray TG, Aziz-Sultan MA, Schefler AC, Wolfe SQ, Hess D, Fernandes CE, Dubovy SR: Supraselective intra-arterial chemotherapy: evaluation of treatment-related complications in advanced retinoblastoma. Clin Ophthalmol 2011;5:171-176.

13 Shields CL, Manjandavida FP, Lally SE, Pieretti G, Arepalli SA, Caywood EH, Jabbour P, Shields JA: Intra-arterial chemotherapy for retinoblastoma in 70 eyes: outcomes based on the International Classification of Retinoblastoma. Ophthalmology 2014;121:1453-1460.

14 Murphree AL: Intraocular retinoblastoma: the case for a new group classification. Ophthalmol Clin North Am 2005;18:41-53. 
15 Shields JA, Shields CL, Meadows AT: Chemoreduction in the management of retinoblastoma. Am J Ophthalmol 2005;140:505-506.

16 Shields CL, Mashayekhi A, Au AK, Czyz C, Leahey A, Meadows AT, Shields JA: The International Classification of Retinoblastoma predicts chemoreduction success. Ophthalmology 2006;113:2276-2280.

17 Shields CL, Shields JA: Basic understanding of current classification and management of retinoblastoma. Curr Opin Ophthalmol 2006; 17:228-234.

18 Chung CY, Medina CA, Aziz HA, Singh AD: Retinoblastoma: Evidence for stage-based chemotherapy. Int Ophthalmol Clin 2015;55: 63-75.

19 Okimoto S, Nomura K: Clinical manifestations and treatment of retinoblastoma in Kobe children's hospital for 16 years. J Pediatr Ophthalmol Strabismus 2014;51:222-229.

20 Shah PK, Narendran V, Kalpana N: Outcomes of intra- and extraocular retinoblastomas from a single institute in South India. Ophthalmic Genet 2015;36:248-250.

21 Naseripour M, Nazari H, Bakhtiari P, Modarres-Zadeh M, Vosough P, Ausari M: Retinoblastoma in Iran: outcomes in terms of patients' survival and globe survival. $\mathrm{Br} \mathrm{J}$ Ophthalmol 2009;93:28-32.

22 Chung SE, Sa HS, Koo HH, Yoo KH, Sung KW, Ham DI: Clinical manifestations and treatment of retinoblastoma in Korea. Br J Ophthalmol 2008;92:1180-1184.

23 Berry JL, Jubran RB, Lee TC, Murphree AL, Lee D, Kim JW: Low-dose chemoreduction for infants diagnosed with retinoblastoma before 6 months of age. Ocul Oncol Pathol 2015; 1:103-110.

24 Schulz KF, Altman DG, Moher D: CONSORT 2010 statement: updated guidelines for reporting parallel group randomized trials. Ann Intern Med 2010;152:726-732

25 Moher D, Hopewell S, Schulz KF, Montori V, Gøtzsche PC, Devereaux PJ, Elbourne D, Eg- ger M, Altman DG: CONSORT 2010 explanation and elaboration: updated guidelines for reporting parallel group randomised trials. $\mathrm{Br}$ Med J 2010;340:c869.

26 Kingston JE, Hungerford JL, Madreperla SA, Plowman PN: Results of combined chemotherapy and radiotherapy for advanced intraocular retinoblastoma. Arch Ophthalmol 1996;114:1339-1343

27 Rodriguez-Galindo C, Wilson MW, Haik BG, Merchant TE, Billups CA, Shah N, Cain A, Langston J, Lipson M, Kun LE, Pratt CB: Treatment of intraocular retinoblastoma with vincristine and carboplatin. J Clin Oncol 2003;15;21:2019-2025.

28 Bartuma K, Pal N, Kosek S, Holm S, All-Ericsson C: A 10-year experience of outcome in chemotherapy-treated hereditary retinoblastoma. Acta Ophthalmol 2014;92:404411.

29 Alkofide A, Ayas M, Khafagah Y, Rawashde A, Anas M, Barria M, Siddiqui K, Almesfer S, Alkatan $\mathrm{H}$ : Efficacy of vincristine and carboplatin as chemo-reduction for advanced bilateral retinoblastoma, the Saudi experience. Saudi J Ophthalmol 2013;27:193-196.

30 Chantada GL, Fandino AC, Raslawski EC, Manzitti J, de Davila MT, Casak SJ, Scopinaro MJ, Schvartzman E: Experience with chemoreduction and focal therapy for intraocular retinoblastoma in a developing country. Pediatr Blood Cancer 2005;44:455-460.

31 Dunkel IJ, Lee TC, Shi W, Beaverson KL, Novetskky D, Lyden D, Finlay JL, McCormick B, Abramson DH: A phase II trial of carboplatin for intraocular retinoblastoma. Pediatr Blood Cancer 2007;49:643-648.

32 Peylan-Ramu N, Bin-Nun A, Skleir-Levy M, Bibas A, Koplewitz B, Anteby I, Pe'er J: Orbital growth retardation in retinoblastoma survivors: work in progress. Med Pediatr Oncol 2001;37:465-470.

33 ImhofSM, Mourits MP, Hofman P, Zonneveld FW, Schipper J, Moll AC, Tan KE: Quantifi- cation of orbital and mid-facial growth retardation after megavoltage external beam irradiation in children with retinoblastoma. Ophthalmology 1996;103:263-268.

34 Turaka K, Shields CL, Meadows AT, Leahey A: Second malignant neoplasms following chemoreduction with carboplatin, etoposide, and vincristine in 245 patients with intraocular retinoblastoma. Pediatr Blood Cancer 2012;59:121-125.

35 Gombos DS, Hungerford J, Abramson DH, Kingston J, Chantada G, Dunkel IJ, Antoneli CB, Greenwald M, Haik BG, Leal CA, Medina-Sanson A, Schefler AC, Veerakul G, Wieland R, Bornfeld N, Wilson MW, Yu CB: Secondary acute myelogenous leukemia in patients with retinoblastoma: is chemotherapy a factor? Ophthalmology 2007;114:13781383.

36 Fontanesi J, Parham DM, Pratt C, Meyer D: Second malignant neoplasms in children with retinoblastoma: the St. Jude Children's Research Hospital experience. Ophthalmic Genet 1995;16:105-108.

37 Araki Y, Matsuyama Y, Kobayashi Y, Toyokawa S, Inoue K, Suzuki S, Makimoto A: Secondary neoplasms after retinoblastoma treatment: retrospective cohort study of 754 patients in Japan. Jpn J Clin Oncol 2011;41: 373-379.

38 Roarty JD, McLean IW, Zimmerman LE:. Incidence of second neoplasms in patients with bilateral retinoblastoma. Ophthalmology 1988;95:1583-1587.

39 Kleinerman RA, Tucker MA, Tarone RE, Abramson DH, Seddon JM, Stovall M, Li FP, Fraumeni JF Jr: Risk of new cancers after radiotherapy in long-term survivors of retinoblastoma: an extended follow-up. J Clin Oncol 2005;23:2272-2279.

40 Abramson DH, Frank CM: Second nonocular tumors in survivors of bilateral retinoblastoma: a possible age effect on radiation-related risk. Ophthalmology 1998;105:573-580. 